\title{
Los Angeles - Entwicklung und Probleme einer Metropole
}

\author{
Harold Haefner
}

\section{Einleitung}

Im Jahre 1781 gründeten die Spanier «El Pueblo de Nuestra Señora la Reina de Los Angeles de Porciuncula». Als Kalifornien 1848 Teil der Vereinigten Staaten wurde, war Los Angeles noch ein kleines, verschlafenes Nest von kaum 1500 Einwohnern. Seither - in einem Zeitraum von nur $120 \mathrm{Jah}$ ren - hat die Stadt einen phantastischen, kaum überseh- und erfaßbaren Aufschwung zu einer der größten und dynamischsten Metropolen der Welt genommen, eine Entwicklung, die in atemraubendem, unaufhaltsamem Tempo weitergeht. Im Buche von Wagner: "Los Angeles», 1935, sind die naturgeographischen Grundlagen und der historische Werdegang anschaulich dargestellt. In zeitlich und thematisch noch weitergestrecktem Rahmen befaßt sich die Artikelserie "Man, Time and Space in Southern California» (Thomas, Edit.) 1959, mit der Entwicklung Südkaliforniens. Darauf aufbauend, soll in der vorliegenden Arbeit versucht werden, die besonders rasante Nachkriegsentwicklung aufzuzeichnen und auf die spezifischen Probleme einzutreten, die sich einerseits aus diesem ungeheuren Wachstum und andererseits aus den besonderen Standortverhältnissen für die Stadt ergeben haben. Der Verfasser dankt dem American Council of Learned Societies, New York, für ein 21monatiges Stipendium an der University of California, Los Angeles, und dem dortigen Department of Geography für die großzügige Gastfreundschaft und tatkräftige Unterstützung. Die Ausführung der Figuren besorgte in verdankenswerter Weise Herr J. Sauter, Assistent am Geographischen Institut der Universität Zürich.

\section{Statistische Stadtbegriffe}

Was versteht man eigentlich unter "Los Angeles»? Wie groß ist die Stadt und wie ist die Agglomeration abzugrenzen? Darüber gehen die Meinungen weit auseinander, und entsprechend unterschiedlich sind die verwendeten Bevölkerungszahlen. Die amerikanische Statistik kennt eine ganze Reihe von Begriffen, die sie im Laufe der letzten Jahrzehnte definierte, um die sich schnell ausdehnenden städtischen Komplexe zu erfassen. Für die aufgrund formaler und funktionaler Gesichtspunkte aufge- stellten Definitionen wird auf Murphy, 1966, Kap. 2 , verwiesen. An dieser Stelle sollen nur die Verhältnisse für unser Untersuchungsobjekt kurz besprochen werden:

\subsection{City of Los Angeles}

Das inkorporierte Stadtgebiet hat eine sehr merkwürdige Form (Fig. 1), die in der historischen Entwicklung begründet liegt. Auffallend sind der lange Korridor gegen S, der der Stadt direkten Anteil am Hafen von Long Beach-Wilmington sichert und die große Flächenausdehnung im N, im San Fernando Valley. Auch bekannte Namen wie Hollywood, Watts usw. sind Teile der eigentlichen Stadt. 1964 betrug die Bevölkerung der City of Los Angeles 2690000 (Fig. 2), für 1970 lautet die Prognose auf 2,9 Millionen 1 .

Der Stadtkomplex ist natürlich längst über diese Grenzen hinausgewachsen. Ein breiter Kranz von inkorporierten Städten (Fig. 1) umgibt die City of Los Angeles, zu denen u. a. Long Beach, Anaheim, Santa Ana, Huntington Beach, Glendale, Garden Grove, Pasadena, Ontario, Burbank, Whittier, Inglewood, Santa Monica, Beverly Hills usw. gehören (allein im Los Angeles County gibt es 75).

Ferner kommt noch überbautes, nicht inkorporiertes County-Areal hinzu. Zusammen mit der City of Los Angeles bilden diese Gebiete eine formale wie funktionale Einheit mit lückenloser Úberbauung. Zur Erfassung dieser Agglomeration müssen darum andere statistische Begriffe angewendet werden.

\subsection{Standard Metropolitan Statistical Area (SMSA)}

Ein SMSA wird aus einem County (oder mehreren Counties) gebildet, das eine Stadt von mindestens 50000 Einwohnern aufweist (Murphy, S. 18 ff.). In unserem Falle besteht das SMSA aus dem Los Angeles County. Der offizielle Name lautet "Los Angeles-Long Beach SMSA", die Einwohnerzahl betrug 19656878200 , für 1970 belaufen sich die Schätzungen auf 7,65 Millionen. Dies ist die üblicherweise für Groß-Los Angeles angegebene Bevölkerungszahl, die aber nicht mit den Realitäten übereinstimmt. 


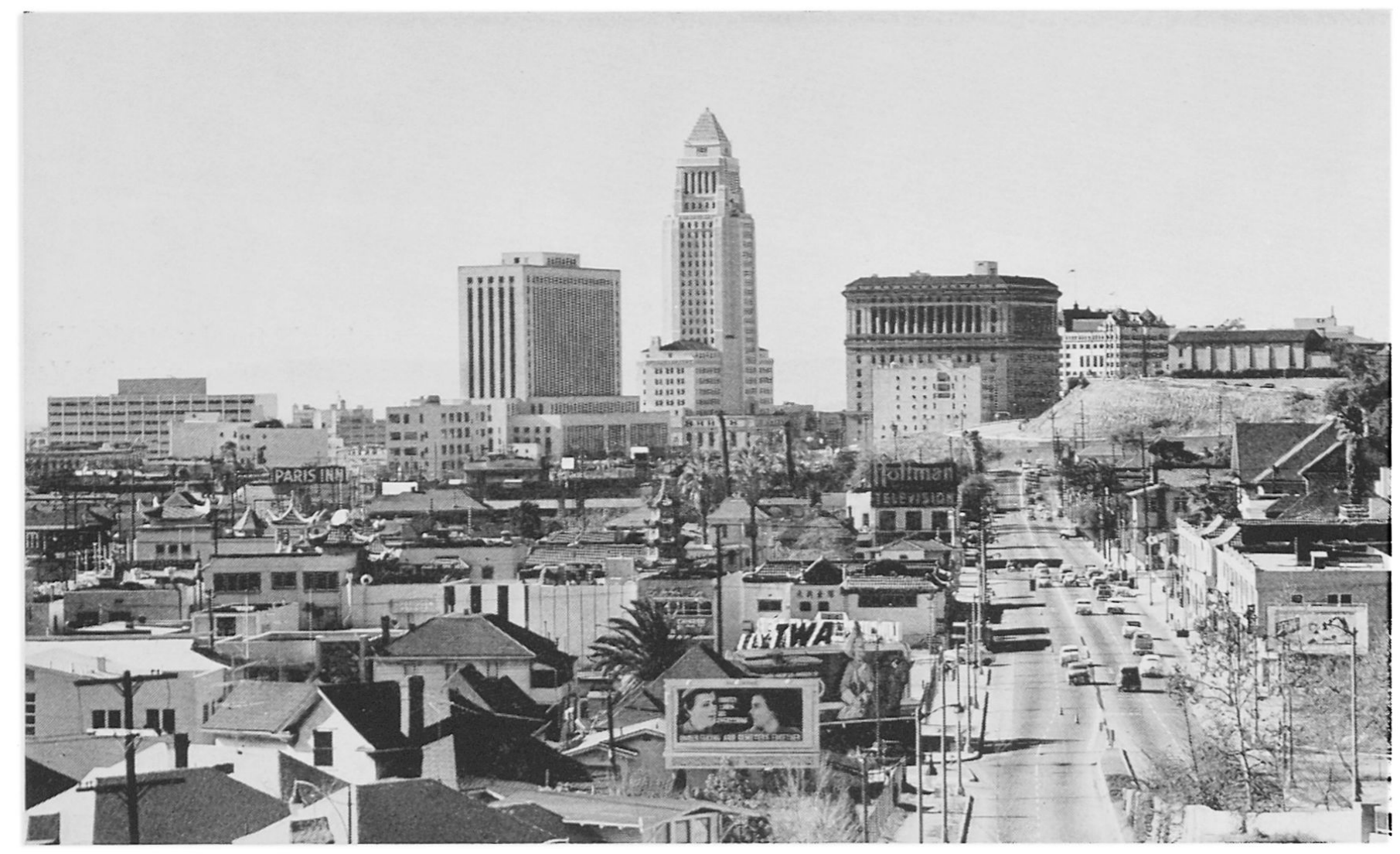

Abbildung 1

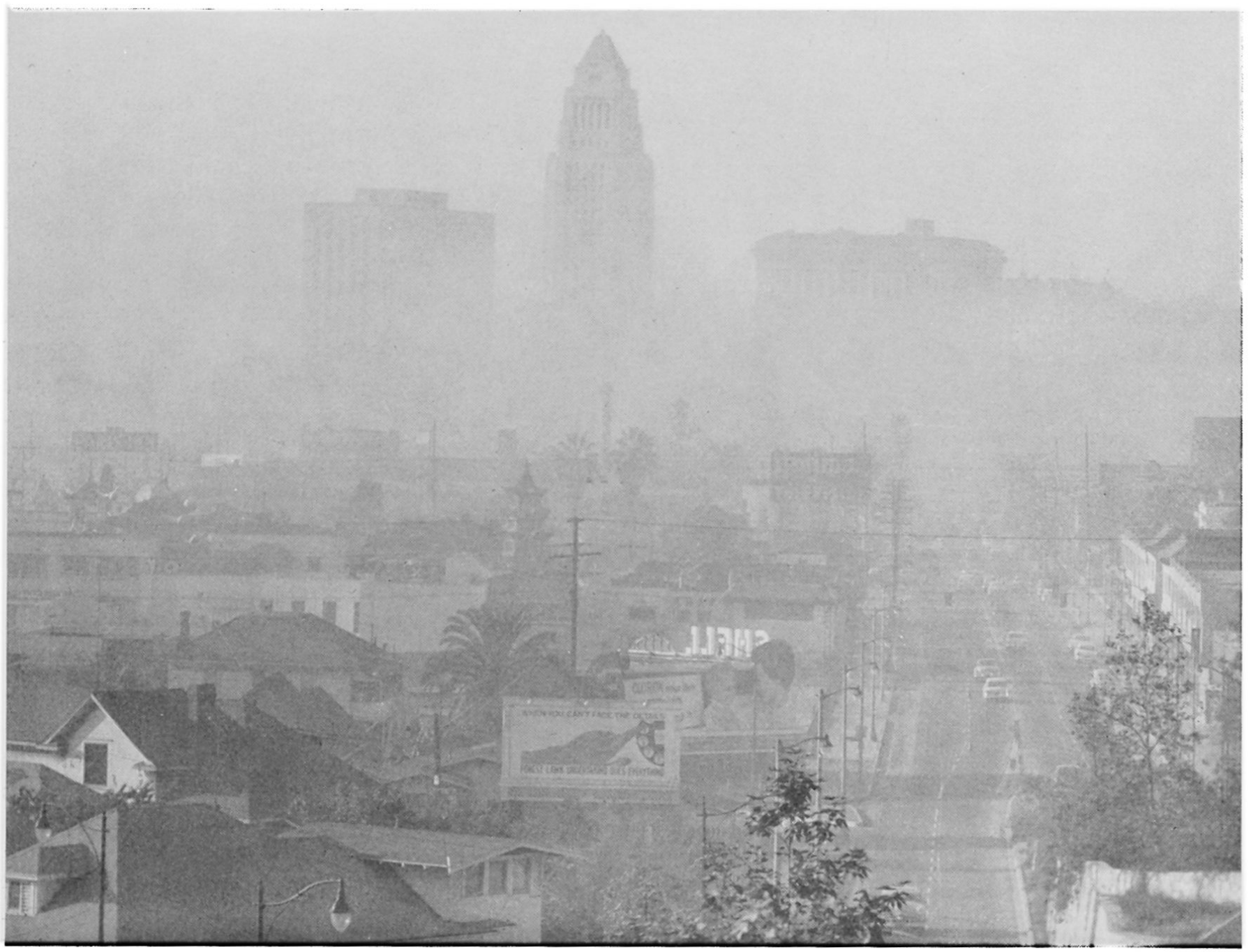

Abbildung 2 


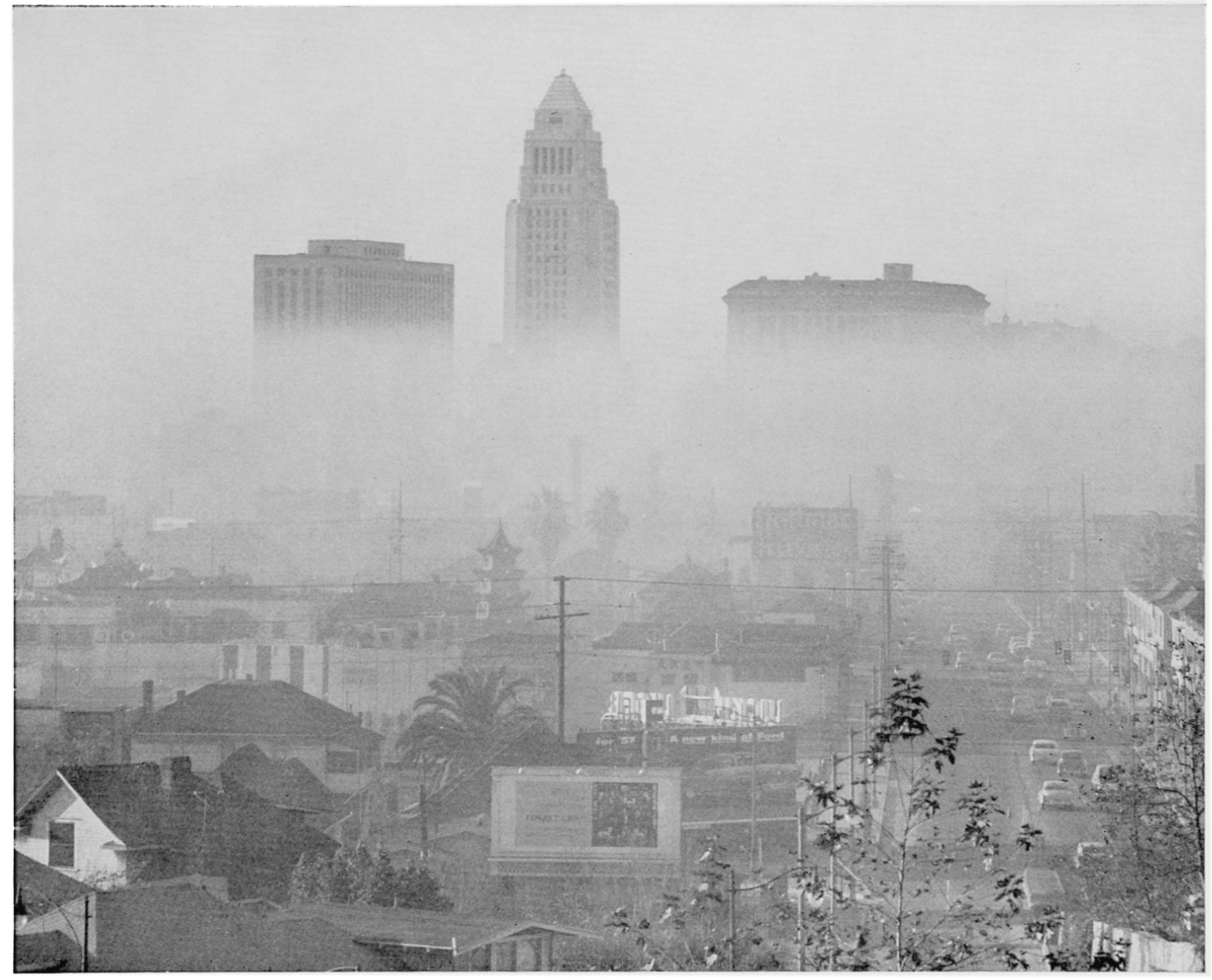

Abbildung 3

Abbildungen 1 bis 3. Smog-Konditionen über Los Angeles.

Downtown Los Angeles von Osten, mit der $138 \mathrm{~m}$ hohen City Hall (Aufnahmen freundlicherweise zur Verfügung gestellt durch Air Pollution District, Los Angeles)

Abbildung 1. Aufnahme an einem klaren Tag ohne Smog

Abbildung 2. Vom gleichen Standort aus bei ausgesprochenen Smog-Verhältnissen mit relativ hochliegender Untergrenze der Inversionsschicht (ca. $300 \mathrm{~m}$ ü. M.)

Abbildung 3. Verhältnisse bei extrem niedrig liegender Untergrenze der Inversionsschicht (weniger als 100 m. ü. M.)

Einerseits schließt der an politische Grenzen gebundene Begriff des SMSA auch Gebiete mit ein, die nicht mehr zur Agglomeration gehören (z. B. die nördlich der San Gabriel Mountains und des Angeles National Forest), auch wenn sie anteilsmäßig kaum ins Gewicht fallen.

Andererseits reicht das vorhin umrissene Gebiet der geschlossenen städtischen Überbauung auch in die benachbarten Counties (Orange, San Bernardino,
Riverside) hinein (Fig. 1), die aber eigene SMSA bilden. Schuld an dieser komplizierten Situation ist die historische Entwicklung. Das Wachstum der Agglomeration vollzog sich nicht zentral von einer Stadt oder von einigen wenigen Kernen, sondern vielmehr polyzentrisch von vielen kleineren und größeren Siedlungen aus, die durch starke Ausdehnung im Laufe der Zeit vollständig zusammenwuchsen. Deshalb wurden die angrenzenden Coun- 
ties als eigene SMSA aufgefaßt, und diese Gliederung wurde bis heute beibehalten. Folgende SMSA haben an der Metropolis Anteil:

\section{SMSA}

Los Angeles-Long Beach

San Bernardino-Riverside-Ontario

Anaheim-Santa Ana-Garden Grove

Total

\section{3. Standard Consolidated Area (SCA)}

Um städtische Komplexe höchster Ordnung zu erfassen, werden mehrere SMSA zu einem SCA zusammengefaßt $\mathrm{t}^{3}$. Bis heute sind in den USA nur deren zwei ausgeschieden, nämlich «New York-Northeastern New Jersey» und "Chicago-Northwestern Indiana». Die Agglomeration von Los Angeles erfüllt diese Anforderungen aber ebenfalls. Die Schaffung eines SCA ist darum auch beantragt und dürfte nach dem Zensus von 1970 verwirklicht werden. Offen bleibt nur die Frage, wie weit dieses SCA gefaßt werden soll. Soll nur die eigentliche Agglomeration von Los Angeles berücksichtigt werden (wie oben angegeben, bestehend aus 3 SMSA), oder soll das ganze Verstädterungsgebiet Südkaliforniens miteinbezogen werden. Die explosionsartige Bevölkerungszunahme seit dem letzten Weltkrieg führte zu einer fast zusammenhängenden Überbauung der Küstenebenen und der anschließenden Täler entlang dem Pazifik von Santa Barbara im N bis zur mexikanischen Grenze, über eine Distanz von rund $500 \mathrm{~km}$. Wenige unbedeutende Lücken bestehen noch, die die Agglomeration von Los Angeles von jenen San Diegos im Süden und Santa Barbara-Ventura-Oxnard im Norden trennen. Sie dürften aber in nächster Zeit ebenfalls geschlossen werden. Aus dieser Sicht scheint es realistischer, nicht eine Metropole Los Angeles, gebildet durch die drei SMSA, sondern gleich eine Megalopolis Südkalifornien ${ }^{4}$ durch Einschluß der drei anschlieBenden SMSA in einem SCA zusammenzufassen. Es ergäbe sich daraus folgende Zusammenstellung:

\section{SMSA}

Metropolis Los Angeles (3 SMSA)

Oxnard-Ventura (Ventura County)

San Diego (San Diego County)

Total

Einwohner $1965^{5}$

9089000

243100

309900

1200800

10842800

Der Entscheid des Bureau of Census bleibt abzuwarten. Wie immer er auch ausfallen wird, das neue
SCA wird so oder so bezüglich Einwohnerzahl jenes von "Chicago-Northwestern Indiana" übertreffen und in der Hierarchie der amerikanischen Stadtkomplexe den zweiten Rang hinter Groß-New York einnehmen.

\subsection{Urbanized Area (UA)}

SMSA und SCA sind in ihrer durch politische Greinzen bestimmten Form für die Abgrenzung städtischer Gebiete, wie erwähnt, nur bedingt geeignet. Deshalb wurde 1950 ein weiterer Begriff, das UA, in die Statistik eingeführt (Murphy, S. 24 ff.). Da er rein formal definiert ist, erfaßt er die wirklich überbauten Flächen, ohne Rücksicht auf politische Grenzen und auf inkorporiertes oder nichtinkorporiertes Gebiet.

Die Agglomeration von Los Angeles umfaßt wiederum 3 UA.

\section{UA}

Los Angeles-Long Beach

(einschl. Orange C.)

Pomona-Ontario

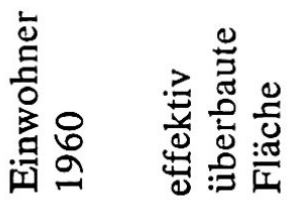

San Bernardino-Riverside

Total

$6488800 \quad 3550 \mathrm{~km}^{2}$

$186500 \quad 184 \mathrm{~km}^{2}$

$377500 \quad 438 \mathrm{~km}^{2}$

$7052800 \quad 4172 \mathrm{~km}^{2}$

Die Zunahme seit 1960 ist ganz bedeutend, die Schätzungen für 1965 lauten bereits auf 8,6 Millionen Einwohner. Die Veröffentlichung einer Karte der überbauten Fläche, auf dem 1960er-Zensus fußend, rechtfertigt sich darum nicht mehr. Hierfür muß der 70er-Zensus abgewartet werden. Eine Vorstellung der überbauten Gebiete gibt hingegen Figur 1, wobei die inkorporierten Städte und das davon eingeschlossene nichtinkorporierte CountyAreal den minimalen Rahmen der Überbauung angeben.

Zusammenfassend sei festgehalten, daß die letztgenannte Zahl von 8,6 Millionen (1965) der städtischen Agglomeration von Los Angeles am besten gerecht wird. Bei Vergleichen ist aber immer die jeweilige statistische Unterlage sorgfältig zu berücksichtigen, und es dürfen die verschiedenartigen Begriffe nicht miteinander vermischt werden.

\section{Die Nachkriegsentwicklung}

Figur 2 veranschaulicht deutlich das außerordentliche Wachstum im 20. Jahrhundert mit dem Boom der 1920er Jahre (Erdöl, Filmindustrie usw.) und dem steilen, unaufhaltsamen Anstieg während 
und nach dem letzten Weltkrieg. Eine ganze Reihe von Gründen ist dafür von ursächlicher Bedeutung, besonders:

a) die politische Situation im pazifischen Raum, die Kalifornien aus einer Randlage plötzlich zur Drehscheibe und Nachschubbasis des ganzen Kriegsgeschehens im Fernen Osten werden ließ. Seither hat sich die Situation nur wenig verändert. Fragen der nationalen Verteidigung, der Korea- und Vietnamkrieg hielten das politische Interesse am Pazifik und damit an der Westküste aufrecht;

b) die Politik der amerikanischen Regierung, die aus den unter a) erwähnten Gründen die Industrie an der Westküste und speziell im Raum von Los Angeles durch riesige Aufträge und Kontrakte förderte. Zuerst betraf dies vor allem den Schiffs- und Flugzeugbau, wofür das erste große Stahlwerk in diesem Raum (Kaiser Steel, Fontana) errichtet wurde. In den Nachkriegsjahren konzentrierten sich die Aufträge vor allem auf die Flugzeug-, Raketen-, Raumfahrt- und Elektronikindustrie;

c) der sich durch diese Förderung ständig vergrößernde Arbeitsmarkt mit den vielen erfolgversprechenden offenen Positionen, der laufend neue $\mathrm{Zu}$ wanderer anzog;

d) die sich entsprechend stark ausweitende Konsumentenbasis, die wiederum zum wesentlichen Standortfaktor für weitere Industrien (z. B. Automobil-, Gebrauchsgüterindustrie usw.) und Dienstleistungsbetriebe aller Art wurde;

e) das milde Klima - publizistisch über den ganzen Kontinent propagiert - das für viele Zuzüger einen zusätzlichen Anreiz bildet, vor allem auch für Pensionierte und Rentner;

f) der immer stärker werdende Trend zur Stadt, speziell zur Großstadt und zur städtischen Lebensform, als allgemeine Zeiterscheinung.

Das Wachstum der Metropole ist also eng mit dem sich rapid ausweitenden Stellenangebot und dem als Folgeerscheinung schnell expandierenden Markt verknüpft. Angezogen von diesem Image der Prosperität und dem günstigen Klima, wandern im Durchschnitt täglich über 1000 Personen in den südkalifornischen Raum ein, davon über zwei Drittel in die eigentliche Metropole. Demgegenüber ist die Auswanderungszahl sehr gering. Hingegen besteht eine ausgesprochen große Mobilität innerhalb des südkalifornischen Raumes.

\section{Die Auswirkungen}

Die charakteristischen Merkmale der formalen und funktionalen Gliederung der Metropole können an dieser Stelle aus Platzgründen leider nicht behandelt werden. Dagegen soll noch auf die Auswirkungen dieser außergewöhnlichen Entwicklung und die sich daraus für die Stadt ergebenden Probleme eingegangen werden. Aus der Vielzahl dieser Problemkreise kann wiederum nur eine kleine Auswahl vorgelegt werden, wobei allerdings die drei aktuellsten, interessantesten, größten, schwierigsten und aufwendigsten zur Sprache kommen sollen, nämlich: Verkehr - Wasser - Luftverunreinigung.

\subsection{Transportsystem}

Das große Wachstum der Stadt fällt im wesentlichen in das Zeitalter des Automobils, was maßgebend dazu beitrug, daß sie sich gänzlich auf dieses Verkehrsmittel ausgerichtet hat. Los Angeles ist die Stadt des Autos. Jedermann ist auf dieses Fahrzeug angewiesen, seine Anzahl darum enorm. 1966 zählte man allein im Los Angeles County 3122 100, in der eigentlichen Metropole etwa 4 Millionen und in ganz Südkalifornien 4865300 Personenwagen ${ }^{6}$.

Der ganzen Region, vor allem aber der Großstadt, fehlt ein öffentliches Massen-Transport-System. Sie hat sich vielmehr dem unrationellsten und platzraubendsten Verkehrsträger, dem Automobil, verschrieben. Die Zahl der Beschäftigten, die die öffentlichen Verkehrsmittel (Bus) benutzen, ist mit etwa 7\% (1960) entsprechend gering 7 . Zu Beginn des 20. Jahrhunderts bis zum Zweiten Weltkrieg bestand allerdings ein gutausgebautes Netz elektrischer Schnellbahnen, das die vielen Ortschaften miteinander verband und zentral auf Downtown Los Angeles ausgerichtet war (Fig. 3). Das immer mehr aufkommende Automobil bedeutete für dieses private Unternehmen eine scharfe Konkurrenz, das seine Rentabilität mit der Zeit in Frage stellte. Die Behörden sahen keine Notwendigkeit zu seiner Unterstützung und Sanierung, so daß allmählich ein Streckenabschnitt nach dem andern stillgelegt wurde, bis die Bahnanlagen total verschwunden waren.

Dafür begann man mit dem Bau eines großzügigen und kostspieligen städtischen Autobahnsystems (freeways), das aber den noch rascher wachsenden Anforderungen trotz ständiger Erweiterungen und Ergänzungen nie völlig genügte und je wird genügen können. Seine jetzige Länge (1967) beträgt im Los Angeles County 570 km, dazu kommen 166 km im Orange County. Das System, nicht mehr auf ein Zentrum ausgerichtet, sondern vielmehr netzartig ausgelegt (Fig. 3), fördert und verstärkt eine schon früh eingeleitete Entwicklung, die Dezentralisierung, in entscheidender Weise.

Los Angeles ist ausgesprochen in die Weite gebaut, mit dominierender Horizontalkomponente. Der vorherrschende Haustyp ist das viel Platz beanspruchende Einfamilienhaus. Entsprechend niedrig sind die Wohn- und Bevölkerungsdichten ${ }^{8}$, die täglich 


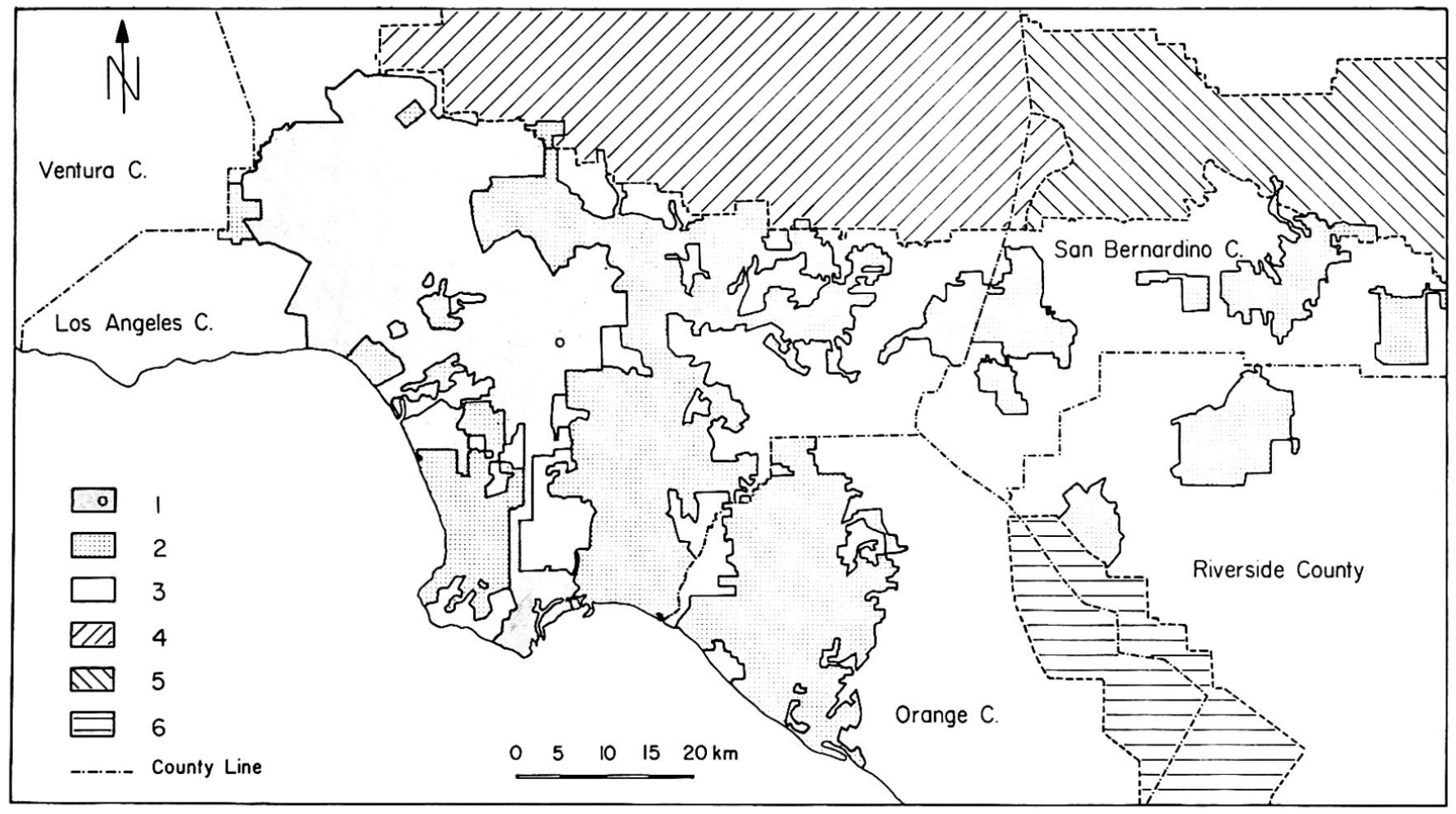

Figur 1. Metropolis Los Angeles: Lage und politische Gliederung. 1 City of Los Angeles mit Stadtzentrum; 2 Weitere inkorporierte Städte; 3 Nicht-inkorporiertes County-Areal; 4 Angeles National Forest; 5 San Bernardino National Forest; 6 Cleveland National Forest

zurückzulegenden Distanzen werden dafür um so größer. Die Industrie, schon seit jeher auf die ganze Küstenebene verstreut, konnte durch das völlige Abstellen auf das Automobil immer peripherere Standorte bevorzugen. Besonders auffallend ist der Trend zur Dezentralisierung bei den Geschäfts- und Einkaufszentren. Downtown Los Angeles hat seine Bedeutung als CBD vollständig verloren. Sein Anteil am Gesamtumsatz der Stadt beträgt keine 5\% mehr. An seine Stelle ist eine Vielzahl größerer und kleinerer Einkaufszentren getreten, sei es in Form langgestreckter Geschäftsstraßen (Foster und Nelson, 1958), verkehrsfreier Ladenstraßen (malls) oder besonders unzähliger, verkehrsmäßig günstig gelegener Shopping Centers aller Größenordnungen. Beim Ausbau und bei der Planung des Verkehrsnetzes bereiten die rechtlichen Grundlagen (wie allgemein bei der Lösung aller in dieser Arbeit besprochenen Probleme) große Schwierigkeiten. Planung und Verwirklichung solcher Aufgaben können nicht mehr innerhalb der Grenzen einer einzigen politischen Organisation allein vorgenommen werden, sondern müssen sich über weiter gefaßte Gebiete mit rechtlich grundverschiedenem Status (inkorp. Städte, Counties usw.) erstrecken. Die Interessen, Ansprüche und die Finanzkraft der einzelnen Stadt- und County-Verwaltungen sind sehr verschieden und folglich nur schwer miteinander zu koordinieren, speziell, wenn auch noch Staats- und Bundesämter ein Mitspracherecht besitzen. Die komplexen politischen Verhältnisse ergeben sich aus der historischen Entwicklung (Fig. 1), wobei nochmals betont werden muß, daß allein in Los Angeles County 75 inkorporierte Städte an der Metropolis Anteil haben. Meistens wird die rechtliche Lösung durch die Schaffung von Zweckverbänden angestrebt. Heute würden die verantwortlichen Behörden ein öffentliches Massen-TransportSystem sehr begrüßen, und sie beschäftigen sich immer wieder mit einer Neuplanung. Schon seit längererer Zeit besteht der "Southern California Rapid Transit District ", der aber nur geringe Fortschritte macht. Generelle Pläne für ein Schnellbahnsystem von $260 \mathrm{~km}$ Länge, teils oberirdisch, teils unterirdisch geführt, wovon in einer ersten Phase ein Linienkreuz von $100 \mathrm{~km}$ erstellt werden soll9, liegen zwar vor, doch selbst die Planungskredite werden nur spärlich zur Verfügung gestellt. So scheint der Gedanke einer Realisierung in nächster Zeit sehr utopisch zu sein. Ebenso fraglich ist es, ob die Bewohner überhaupt von einer Schnellbahn wieder Gebrauch machen würden, ist doch ihr Lebensstil absolut auf das Automobil ausgerichtet. Diese Gewohnheit zu ändern, dürfte mit einigen Schwierigkeiten verbunden sein. Hinzu kommt, daß die dezentralisierten Geschäfts- und Industrieviertel sowie die locker überbauten und weit gestreuten Wohnquartiere nach einem sehr langen und dichten 


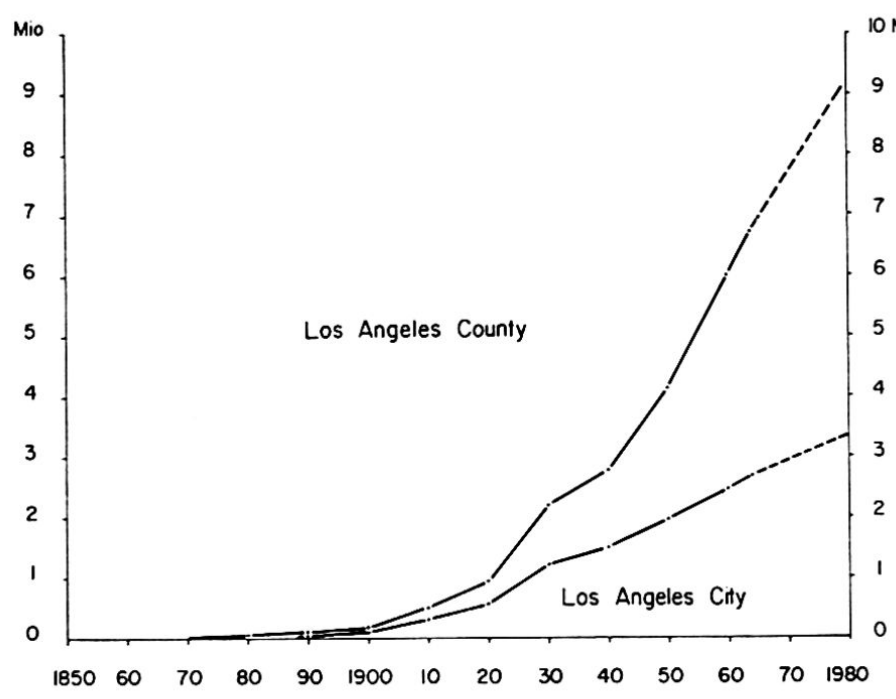

Figur 2. Bevölkerungsentwicklung 1850-1980

Schienennetz mit vielen Stationen verlangen, wodurch aber wiederum Zeitgewinn und Rendite in Frage gestellt würden. So scheint eine Rückkehr zu einem vernünftigen Massentransportmittel unwahrscheinlich, womit zwangsläufig der Ausbau des Autobahnnetzes als einzige Alternative bleibt.

4.2. Wasser

Das semiaride Klima (die Küstenebene hat nach Koeppen ein CS-Klima, das landeinwärts und gegen Süden schnell in BS- und BW-Klimate übergeht) und die jahreszeitlich sehr ungleichmäßig verteilten Niederschläge (sie konzentrieren sich auf wenige Tage im Winter) limitieren, in Kombination mit den geologischen Verhältnissen, die lokalen Wasservorräte. Sie vermögen den Bedarf einer Großstadt nie mehr zu befriedigen. Für die ersten Siedlungen fand sich in den Schottern der Aufschüttungsebene genügend und gutes Grundwasser.

Zur Deckung des rapide anwachsenden Verbrauchs der Metropole und seiner Industrie reichte es bald nicht mehr aus, und das Wasser mußte aus immer größerer Entfernung und unter enormem Kostenaufwand mühsam herbeigeschafft werden.

Heute erhält Los Angeles und mit ihr ganz Südkalifornien Wasser über drei große Aquädukt-Systeme (Fig. 4). Ihre Verteilernetze sind weitgehend zusammengehängt und erstrecken sich bis an die mexikanische Grenze. Die Hauptverteileräste sind ebenfalls in Figur 4 eingetragen.

Das älteste System, der Los Angeles Aqueduct, wurde 1907-1913 von der City of Los Angeles gebaut. Es nutzt die klaren Gebirgsgewässer des Owens River und seiner Nebenäste auf der E-Seite der Sierra Nevada, die ein ausgezeichnetes Trinkwasser abgeben. Das Wasser wird in einer teils offenen, teils geschlossenen Leitung ohne Einschaltung einer einzigen Pumpe über eine Distanz von $377 \mathrm{~km}$ in ein Reservoir im San Fernando Valley geleitet. Um den ständig wachsenden Bedarf zu decken, wurde das System allmählich immer weiter nordwärts bis ins Becken des Mono Lake ausgedehnt. Seine gesamte Länge beträgt jetzt rund $550 \mathrm{~km}$, wobei zum Teil auch das natürliche Flußbett für den Transport benutzt wird. Zahlreiche Stauseen speichern die Frühlingsschmelzwasser und regulieren den Abfluß. Das Vorgehen der City of Los Angeles, um in den Besitz des Wassers zu gelangen, war einfach und brutal. Sie kaufte sukzessive alles Land entlang dem Owens River und seiner Nebenbäche, vor allem das wertvollste bewässerbare Land, womit sie in den Besitz der dazugehörenden Wasserrechte gelangte. $\mathrm{Da} \beta$ dadurch einem ganzen, landwirtschaftlich orientierten Tal, dem Owens Valley, die Existenzgrundlage entzogen wurde, sei nur am Rande erwähnt (Baugh, 1937). Die Stadt besitzt heute im Inyo- und Mono-County 122000 ha Land, das heißt praktisch alles Kulturland im Einzugsbereich des Owens River und Mono Lake. Die tägliche Förderleistung des Aquädukts beträgt maximal etwa 11 Millionen Hektoliter. Gegenwärtig wird das ganze System um eine zweite Leitung erweitert, wodurch die Kapazität um $48 \%$ gesteigert werden kann. Das notwendige Wasser soll durch zusätzliches Umleiten von Wasser aus dem Mono Basin und durch vermehrtes Pumpen von Grundwasser im Owens Valley gewonnen werden.

Gleichwohl genügten die verfügbaren Mengen den Ansprüchen der wachsenden Großstadt schon nach kurzer Zeit nicht mehr. Die Suche nach Wasser begann erneut. Diesmal wandte man sich dem Colorado River zu. Der 1932-1941 erbaute Colorado Aqueduct bringt täglich bis zu 40 Millionen Hektoliter Wasser vom Lake Havasu hinter dem Parker Dam über eine Distanz von $400 \mathrm{~km}$ in ein Reservoir bei Riverside. Die für die Pumpen notwendige Energie stammt mehrheitlich von den Kraftwerken am Hoover Dam. An diesem Projekt beteiligte sich die City of Los Angeles nicht mehr allein, sondern schloß sich mit andern interessierten Städten zum sogenannten Metropolitan Water District of Southern California zusammen. Heute sind insgesamt 117 Städte an diesem Verband beteiligt. So erstaunt es nicht, daß das am Aquädukt angeschlossene Hauptverteilernetz eine Länge von über $700 \mathrm{~km}$ hat. Das sprunghafte Ansteigen der Bevölkerung in den Nachkriegsjahren und die durch das Bundesgericht verfügte Reduktion des Kalifornien zustehenden Anteils an Colorado-Wasser 10 zwang zu noch viel gigantischeren Bauplänen. Um die nötigen Wasser.. mengen zu finden, wurde ein Projekt entworfen, dessen Einzugsgebiet bis in die nördliche Sierra Nevada reicht. Die Verwirklichung erfolgte im Rahmen des großange!egten California State Water Projects, zum Teil sogar unter Beizug des Bundes ${ }^{11}$. Am Feather River wurde ein mächtiger Damm (Oroville Dam) errichtet. Von dort fließt das Wasser zuerst auf natürlichem Wege ins Delta-Gebiet, einer 


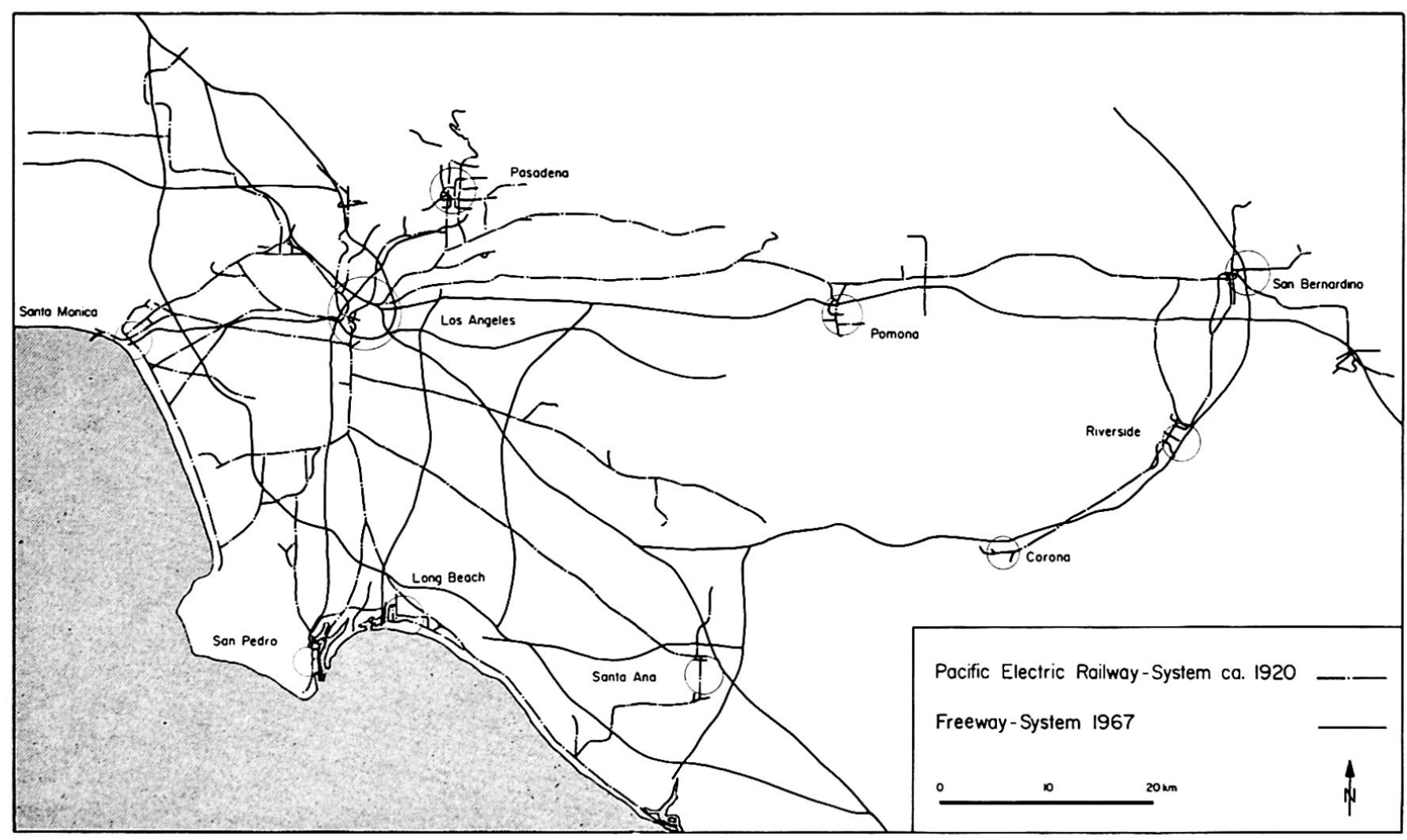

Figur 3. Früheres Schienen- und heutiges Autobahnsystem

Sumpf- und Polderlandschaft im Mündungsbereich des Sacramento- und San Joaquin River. An dessen Südende beginnt der neue California Aqueduct. Uber diese gigantische Wasserstraße12 wird das Wasser praktisch aus Meeresniveau $700 \mathrm{~km}$ weit südwärts gepumpt, zuerst ins obere San Joaquin Valley und dann über die $1000 \mathrm{~m}$ hohen Tehachapi Mountains nach Südkalifornien. Die Transportmenge beträgt im Tag 67,6 Millionen Hektoliter, die Hälfte davon steht dem Metropolitan Water District of Southern California zur Verfügung, der dafür ungefähr zwei Drittel der Bau- und Betriebskosten zu bezahlen hat. Der Rest dient zur Belieferung zahlreicher Küstenabschnitte und der Bewässerung der besonders trockenen Westseite des San Joaquin Valley sowie weiterer Gebiete entlang dem Aquädukt. Die dadurch hervorgerufenen Veränderungen der Kulturlandschaft werden bedeutend sein (Haefner, 1968). Der nördliche Abschnitt des Systems ist bereits in Betrieb, bis 1972 wird die ganze Anlage fertiggestellt sein. Damit hofft man, den Bedarí der Metropolis bis zum Jahr 2000 decken zu können.

\section{3. $S m o g$}

Das wohl schwierigste und kostspieligste Problem erwuchs der Agglomeration aus den spezifischen atmosphärischen Verhältnissen in Kombination mit ihrer topographischen Lage.

Im Sommerhalbjahr liegt Südkalifornien im Bereich des subtropischen Hochdruckgürtels mit Tendenz zu absinkender, trockener Luft in der Höhe. Die Bodenschicht dagegen besteht aus relativ kühler, maritimer Luft, die durch die Meerbrise tagsüber landeinwärts bewegt wird (Fig. 5). So kommt es über der Ebene von Los Angeles häufig zur Ausbildung einer Inversionsschicht, die ein Entweichen der über der Stadt lagernden Luft nach oben unmöglich macht. Ebenso kann sie der umliegenden Bergketten (Fig. 5) wegen nicht ins Innere abziehen. Da die nächtliche Ausgleichsbewegung der Luft Richtung Meer weniger stark ist als die Meerbrise des Tages $(8-12 \mathrm{~km} / \mathrm{h}$ gegenüber $2-5 \mathrm{~km} / \mathrm{h}$, nach Reith, 1951), erfolgt auch kein Abfließen in dieser Richtung.

Die Luft bleibt also für einige Zeit über der Stadt stationär und wird mit Abgasen und Staubpartikeln stark angereichert, was zur Bildung des bekannten $S m o g$ führt. Täglich entweichen allein in Los Angeles County 14610 Tonnen ${ }^{13}$ Abgase in die Luft, vorwiegend Kohlenwasserstoffe und andere organische Gase (2700 t), $\mathrm{NO}_{\mathrm{x}}(920 \mathrm{t}), \mathrm{SO}_{2}(630 \mathrm{t})$, CO (10 220 t) und Staubpartikel aller Art (140 t). Hauptquellen der Luftverschmutzung sind die Auspuffgase der Automobile, nämlich zu $85 \%$ (bei $\mathrm{CO}$ zu $98 \%$ ), die Industrien, speziell die Raffinerien 


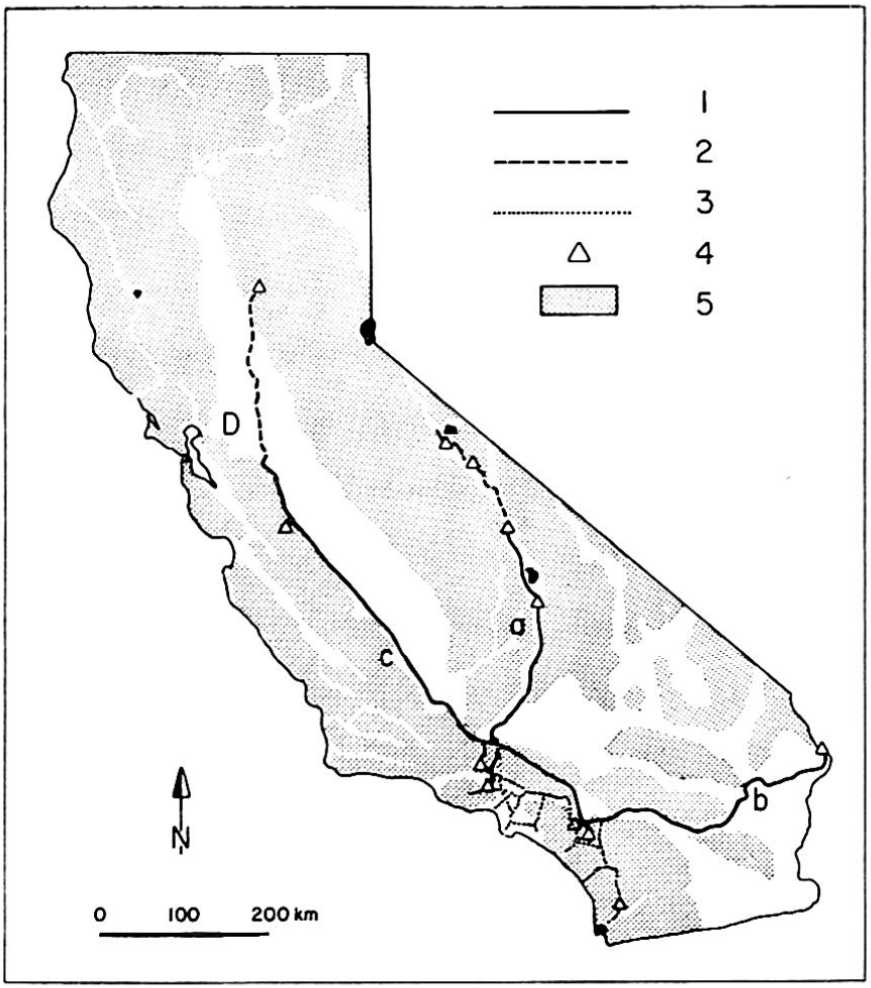

Figur 4. Die Wasserversorgung von Los Angeles. a Los Angeles Aqueduct; b Colorado Aqueduct; c California Aqueduct.

1 Künstliche Wasserleitungen (Kanäle, Röhren);

2 Benützung des natürlichen Flußbettes; 3 Hauptverteilersystem; 4 Staudämme und Stauseen;

5 Hügel und Gebirge

$(3 \%)$ und chemischen Fabriken (1\%) sowie die Heizungen der Gebäude zu $6 \%$ (bei $\mathrm{SO}_{2}$ zu $78 \%$ ). Mit aller Deutlichkeit zeigt sich ein weiterer großer Nachteil des Automobils gegenüber einem «sauberen» elektrischen Schnellbahnsystem. Äußerst schädlich und unangenehm wirkt sich die zu hohe Anreicherung von Ozon aus, das sich bei relativ stationärer Luft durch photochemische Reaktion aus den Abgasen bilden kann. Alle Voraussetzungen hierzu sind speziell im Sommerhalbjahr wiederholt gegeben: Die Inversionsschicht, genügend Sonnenlicht und die relativ unbewegte Luft. Letztere stellt sich vorab im früheren Vormittag ein, in der Ubergangsphase von der Land- zur Seebrise, die ausgerechnet mit der morgendlichen "rush-hour», während der die Verkehrsfrequenzen besonders hoch sind, zusammenfällt. Los Angeles' Smog ist also eine ausgesprochene Erscheinung der warmen Jahreszeit (spez. Juni-November), im Gegensatz zum Beispiel zum Londoner Smog. Im Laufe des Tages erfolgt eine starke Zunahme der Luftverunreinigung mit einer ausgeprägten Spitze am späten Nachmittag. Die schlimmsten Stunden sind etwa zwischen 2-5 Uhr. Die Luft wird deutlich getrübt, ein gelb- lich-weißer Schleier lagert über der Stadt, die Sicht ist merklich reduziert (Abb. 1-3). Die Folgen davon sind Pflanzenschädigungen und beim Menschen Reizerscheinungen der Augen, Kopfschmerzen usw. Die Empfindlichkeit gegenüber Smog ist selbstverständlich individuell sehr verschieden. Wie weit der Mensch davon gesundheitlich betroffen werden kann, ist noch nicht hinreichend untersucht. Der bisherige Verunreinigungsgrad gilt nach wie vor als ungefährlich. Am stärksten vom Smog betroffen werden die Teile über den am dichtesten bebauten Gebieten, die ringförmig um Downtown Los Angeles liegen, in einem Radius von etwa 30 km (Fig. 5). Die Meerbrise drückt die verunreinigte Luft landeinwärts an die Hügel und in die Canyons hinein (z. B. gegen die als beste Wohnlage geltenden Hänge von Beverly Hills und Bel Air) und durch die schmalen Durchgänge ins San Fernando-, San Gabrielund Pomona-Valley usw. Eventuell erfolgt ein langsames Abfließen über die niedrigen Pässe Richtung Wüste. Die vom Smog besonders stark befallenen Gebiete haben sich mit der zunehmend wachsenden Agglomeration nach und nach ausgedehnt, vor allem Richtung Süden und gegen Orange County. Trotzdem trägt bis heute allein das Los Angeles County die Kontrolle und Bekämpfung der Luftverschmutzung (L. A. County Air Pollution Control District) mit einem jährlichen Budget von etwa 3,5 Millionen Dollar (1966-1967).

Erstmals realisierte man die Unannehmlichkeiten des Smogs in den 1940er Jahren. Die Anzahl SmogTage nahm daraufhin sehr schnell zu. 1947 bereits wurde der "Control-District» ins Leben gerufen. Die Quellen der Luftverunreinigung haben seither einen starken Wandel mit betonter Verlagerung zum Automobil hin erfahren. Geblieben sind die immer größer werdenden Mengen an Schmutzstoffen. Die stationären Quellen konnten in den letzten Jahrzehnten weitgehend unter Kontrolle gebracht werden. Alle Unternehmen, die irgendwie Luftverunreinigung verursachen, bedürfen einer speziellen Bewilligung. Die Vorschriften sind streng, so daß bis 1967 allein für Abgas- und Staubauffanggeräte 135 Millionen Dollar aufgebracht werden mußten (ohne Entwicklungs- und Unterhaltskosten). Der Effekt war sehr positiv, obwohl er immer wieder durch die wachsende Zahl der Automobile verwischt wurde. Diese wirksam zu kontrollieren ist weit schwieriger. Wohl werden die Vorschriften immer strenger und umfassender, und für jedes zugelassene Auto werden Abgasgeräte vorgeschrieben (Smog devices). Ihr richtiges Funktionieren läßt sich hingegen nur schwer kontrollieren.

Die Luftverunreinigungen werden laufend gemessen und registriert. Beim Überschreiten kritischer Grenzwerte wird die Smog-Warnung (smog-alert) ausgelöst und über alle Kommunikationsmittel verbreitet. Es besteht folgendes Warnschema: 


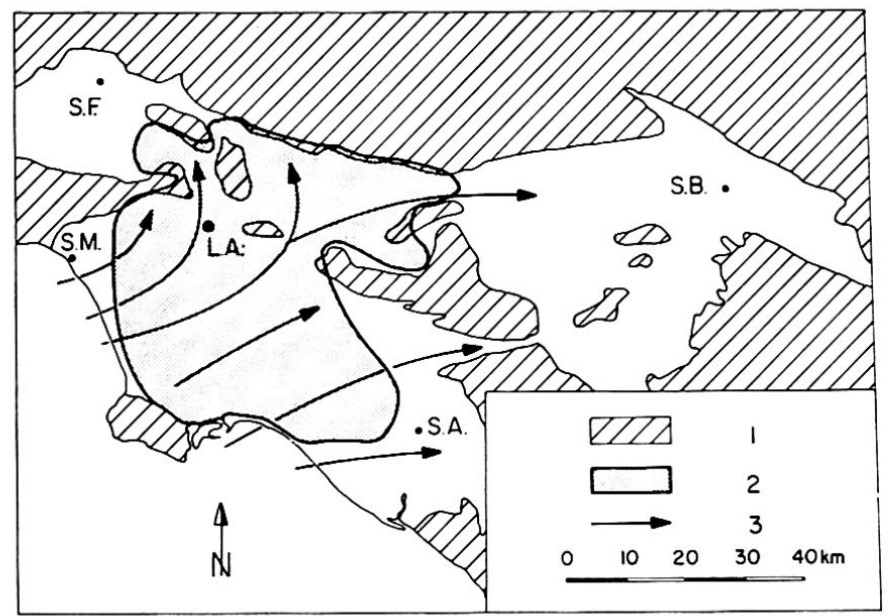

Figur 5. Luftverunreinigung über der Küstenebene von Los Angeles (nach Reith, 1951, leicht abgeändert). 1 Höhenzüge, 2 Gebiete, die am stärksten vom Smog betroffen werden; 3 Hauptrichtung der Meerbrise (tagsüber)
Alarmstufen

Anteil je Millionen Luftteilchen $\begin{array}{llll}\mathrm{CO} & \mathrm{NO}_{\mathrm{x}} & \mathrm{SO}_{2} & \text { Ozon }\end{array}$

1. Alarmstufe bei

100

200

2. Alarmstufe bei

300

$\begin{array}{rrl}3 & 3 & 0,5 \\ 5 & 5 & 1 \\ 10 & 10 & 1,5\end{array}$

1. Alarmstufe: Konzentration nahe der maximal tolerierbaren Menge, Sicherheit noch gewährleistet, aber Präventivmaßnahmen erforderlich.

2. Alarmstufe: Gesundheitsgefährdung besteht in einleitendem Stadium.

3. Alarmstufe: Konzentration mit ernsthafter Gesundheitsgefährdung.

Jährlich müssen bis zu 15 Smogwarnungen (1.Stufe) ausgelöst werden, im Durchschnitt 5, wobei seit den 50er Jahren eine leichte Abnahme zu verzeichnen ist. Bei Alarm müssen gewisse Industrien ihre Produktion einstellen oder auf eine "saubere» Energiequelle (z. B. Erdgas) umschalten. Die Bevölkerung wird über Massenmedien ersucht, alle nicht absolut notwendigen Autofahrten einzustellen. Alle Smogwarnungen erfolgten bis jetzt wegen zu hoher Ozonkonzentration. Die zweite Alarmstufe wurde noch nie erreicht. Die höchste gemessene Ozon-Konzentration betrug 0,9 Teile je Millionen Luftteilchen (13. September 1955). $\mathrm{NO}_{\mathrm{x}}$-Verbindungen erreichten zweimal beinahe die Alarmlimite.

Die Stärke und Dauer einer Smogsituation hängt ganz von den meteorologischen Bedingungen ab. Besonders wichtig ist die Untergrenze der Inversionsschicht. Je tiefer die Inversionsschicht, desto schlimmer die Verhältnisse (Abb. 1-3). In der Regel liegt ihre Untergrenze bei etwa $300 \mathrm{~m}$ ü. M., sie kann aber bis auf $100 \mathrm{~m}$ ü. M. absinken. Bleiben die atmosphärischen Verhältnisse stabil und bildet sich die Inversionsschicht täglich neu, so kann sich eine "smog-attack» über mehrere Tage erstrecken. Die längste dauerte 15 Tage (20. 11 - 4. 12. 1949).

Die Behörden sind relativ optimistisch und glauben, mit strikten Vorschriften auch die Hauptquelle, die Automobilabgase, unter Kontrolle zu bringen. $\mathrm{Ob}$ allerdings eine fühlbare Verbesserung in den nächsten Jahren erreicht werden kann, bleibt fraglich. Solange das Automobil nach dem Prinzip des Verbrennungsmotors funktioniert und nicht auf ein grundsätzlich anderes Antriebssystem umgestellt wird, läßt sich das Smogproblem für Los Angeles (und andere Großstädte, die von den hier vorgenommenen Untersuchungen profitieren) kaum definitiv lösen.

\section{Zukunft}

Die rasante Entwicklung wird dank der anhaltenden Konjunktur weiter fortschreiten, die Bevölkerung in gleichem Umfang zunehmen, wobei vor allem die an Los Angeles County anstoßenden Counties besonders profitieren werden. Die Schätzungen für das Jahr 2000 belaufen sich für Los Angeles County auf 11 Millionen Einwohner, für ganz Südkalifornien auf 24 Millionen. Die hier gestreiften Probleme vergrößern und komplizieren sich entsprechend. Es zeigt sich mit aller Deutlichkeit, daß die Küstenebene von Los Angeles als Standort für eine Metropole naturgeographisch gesehen ungeeignet ist. Die daraus resultierenden Probleme lassen sich nur mit größtem Aufwand und Mitteln erkaufen.

\section{Literaturverzeichnis}

Air Pollution Control District: Air Pollution Data for Los Angeles County, Jan. 1967 (und weitere vervielfältigte Berichte).

Bailey, Harry P.: The Climate of Southern California. Univ. of Calif. Press. Berkeley 1966.

Baugh, Ruth E.: Land Use Changes in the Bishop Area of Owens Valley. Economic Geogr., Vol. 13, 1937, S. 17-34.

California Information Almanac 1966. Lakewood, Calif.

Cooke, R. U. und Simmons, I. G.: Some Recent Changes in California. Tijdschrift voor Econ. en Soc. Geografie, Nov./Dec. 1966.

Durrenberger, Robert W.: Patterns on the Land. Palo Alto 1967.

Foster, G. J. und Nelson, H. J.: Ventura Boulevard - A String-Type Shopping Street; Real Estate Reserarch Program. Bureau of Business and Eco- 
nomic Research, Univ. of Calif., Los Angeles 1958. Gottmann, J.: Megalopolis - The Urbanized Northern Seaboard of the United States. Cambridge, Mass., 1961.

Grey, Arthur L.: Los Angeles - Urban Prototype. Land Economics, 35, Aug. 1959, S. 232-242.

Haefner, H.: Wasser für den Süden Kaliforniens. Geographische Rundschau, 10, 1968, S. 381-387. Lantis, David W.: Los Angeles. Focus, Vol. 12, No. 9, 1962.

Lantis, D. W., Steiner, R. und Karinen, A. E.: California - Land of Contrasts. Belmont, Calif., 1963.

Leighton, Philip A.: Geographical Aspects of Air Pollution, Geogr. Review, Vol. 56, No. 2, 1966.

Murphy, R. E.: The American City - An Urban Geography. New York 1966.

Office of Statistical Standards: Standard Metropolitan Statistical Areas. Washington D. C. 1967.

Preston, R. E.: Urban Development in Southern California between 1940 und 1965. Tijdschrift voor Econ. en Soc. Geografie, Vol. 58, No. 5, 1967, S. 237-254.

Reith, J. E.: Los Angeles Smog. Yearbook of the Assoc. of Pacific Coast Geographers, Vol. 13, 1951, p. 24-32.

Steiner, Rodney: Reserved Lands and the Supply of Space for the Southern California Metropolis. Geogr. Review, Vol. 56, No. 3, 1966, p. 344-362. Thomas, W. L. (Edit.): Man, Time and Space in Southern California. Annals, Vol. 49, No. 3, 1959. Time - Life Library of America: The Pacific States. New York.

Wagner, Anton: Los Angeles. Leipzig 1935.

Wolman, Abel: The Metabolism of Cities. Scientific American, Vol. 213, No. 3, 1965.
Anmerkungen

1 Sämtliche Zahlen, soweit nicht anders angegeben, nach U.S. Bureau of Census, 1960, und Preston, 1967.

2 Nach: Office of Statistical Standards, 1967.

${ }^{3}$ Es können zusätzlich auch weitere Counties, die kein SMSA bilden, in einem SCA eingeschlossen werden.

4 Im Sinne des von Gottmann, 1961, definierten Stadtkomplexes für die atlantische NE-Küste der USA.

5 Nach: Office of Statistical Standards, 1967.

6 Briefliche Mitteilung des Automobile Club of Southern California, vom 19. 4. 1967.

7 Als Vergleich: New York 55\% und Chicago 32\% (1960).

8 Die Bevölkerungsdichte ist eine der niedrigsten aller amerikanischer Großstädte; sie beträgt für Los Angeles 1820 Einwohner $/ \mathrm{km}^{2}$, im Vergleich dazu für New York 2850, für Chicago 2400.

9 Los Angeles Time, 24. 6. 1966.

10 Bundesgerichtsentscheid 1964 im Falle Arizona gegen Kalifornien, in dem das jährliche Bezugsrecht Kaliforniens auf Colorado-Wasser eingeschränkt wurde, wovon natürlich auch der Metropolitan Water District betroffen wird.

11 Das U.S. Bureau of Reclamation beteiligt sich am Bau des St.-Louis-Staudamms und -Kanals, einem Teilstück des California Aqueduct im San Joaquin Valley.

12 Ihre durchschnittliche Wasserführung ist größer als jene der Reuß bei der Einmündung in die Aare. 13 Air Pollution Control District, County of Los Angeles; 1967, S. 3. 